\section{OPEN ACCESS}

Edited by:

Tie Liu,

University of Florida, United States

Reviewed by:

Silin Zhong,

The Chinese University of Hong Kong,

China

David Chagne,

The New Zealand Institute for Plant and Food Research Ltd.

New Zealand

*Correspondence:

Sean Myles

sean.myles@dal.ca

tPresent address:

Yihui Gong,

College of Agriculture

and Biotechnology, Hunan University

of Humanities, Science

and Technology, Loudi, China

Specialty section: This article was submitted to Plant Genomics,

a section of the journal

Frontiers in Genetics

Received: 23 February 2021

Accepted: 10 May 2021

Published: 22 June 2021

Citation:

Migicovsky Z, Yeats TH, Watts S,

Song J, Forney CF

Burgher-MacLellan K, Somers DJ,

Gong Y, Zhang Z, Vrebalov J,

van Velzen R, Giovannoni JG,

Rose JKC and Myles S (2021) Apple

Ripening Is Controlled by a NAC

Transcription Factor.

Front. Genet. 12:671300.

doi: 10.3389/fgene.2021.671300

\title{
Apple Ripening Is Controlled by a NAC Transcription Factor
}

\begin{abstract}
Zoë Migicovsky ${ }^{1}$, Trevor H. Yeats 2,3 , Sophie Watts ${ }^{1}$, Jun Song ${ }^{4}$, Charles F. Forney ${ }^{4}$, Karen Burgher-MacLellan ${ }^{4}$, Daryl J. Somers ${ }^{5}$, Yihui Gong ${ }^{6}$, Zhaoqi Zhang ${ }^{6}$, Julia Vrebalov',3, Robin van Velzen ${ }^{7}$, James G. Giovannoni, ${ }^{3,8}$, Jocelyn K. C. Rose ${ }^{2}$ and Sean Myles ${ }^{1 *}$
\end{abstract}

' Department of Plant, Food and Environmental Sciences, Faculty of Agriculture, Dalhousie University, Truro, NS, Canada, ${ }^{2}$ Plant Biology Section, School of Integrative Plant Science, Cornell University, Ithaca, NY, United States, ${ }^{3}$ Boyce Thompson Institute, Cornell University, Ithaca, NY, United States, ${ }^{4}$ Agriculture and Agri-Food Canada, Kentville, NS, Canada, ${ }^{5}$ Vineland Research and Innovation Centre, Vineland Station, ON, Canada, ${ }^{6}$ College of Horticulture, South China Agriculture University, Guangzhou, China, ${ }^{7}$ Biosystematics Group, Wageningen University, Wageningen, Netherlands, ${ }^{8}$ United States Department of Agriculture, Robert W. Holley Center, Cornell University, Ithaca, NY, United States

Softening is a hallmark of ripening in fleshy fruits, and has both desirable and undesirable implications for texture and postharvest stability. Accordingly, the timing and extent of pre-harvest ripening and associated textural changes following harvest are key targets for improving fruit quality through breeding. Previously, we identified a large effect locus associated with harvest date and firmness in apple (Malus domestica) using genome-wide association studies (GWAS). Here, we present additional evidence that polymorphisms in or around a transcription factor gene, NAC18.1, may cause variation in these traits. First, we confirmed our previous findings with new phenotype and genotype data from $\sim 800$ apple accessions. In this population, we compared a genetic marker within NAC18.1 to markers targeting three other firmness-related genes currently used by breeders (ACS1, ACO1, and PG1), and found that the NAC18.1 marker was the strongest predictor of both firmness at harvest and firmness after 3 months of cold storage. By sequencing NAC18.1 across 18 accessions, we revealed two predominant haplotypes containing the single nucleotide polymorphism (SNP) previously identified using GWAS, as well as dozens of additional SNPs and indels in both the coding and promoter sequences. NAC18.1 encodes a protein that is orthogolous to the NON-RIPENING (NOR) transcription factor, a regulator of ripening in tomato (Solanum lycopersicum). We introduced both NAC18.1 transgene haplotypes into the tomato nor mutant and showed that both haplotypes complement the nor ripening deficiency. Taken together, these results indicate that polymorphisms in NAC18.1 may underlie substantial variation in apple firmness through modulation of a conserved ripening program.

Keywords: apple, fruit ripening, NAC-domain transcription factor, apple texture, marker-assisted selection

\section{INTRODUCTION}

Despite their diverse structure, ontogeny, and biochemical composition, fleshy fruits from a taxonomically broad range of species undergo coordinated ripening processes that have many features in common. Ripening involves numerous physiological and biochemical changes that render the fruit attractive and nutritious for consumption by seed-dispersing animals, or human 
consumers in the case of crops. These changes include the accumulation of sugars, pigments, and flavor or aroma compounds, as well as a loss of flesh firmness due in large part to the controlled modification and depolymerization of cell wall polysaccharides (Wang et al., 2018). Processes involved in ripening are regulated by conserved and convergently evolved networks of transcription factors and hormones, such as ethylene in climacteric fruit where a respiratory burst occurs at the beginning of ripening (Lü et al., 2018).

Various aspects of fruit ripening have been particularly well-studied in tomato (Solanum lycopersicum L.) and the characterization of tomato ripening mutants has revealed a regulatory network consisting of transcription factors, hormones, and epigenetic modifications (Giovannoni et al., 2017). Among the best studied ripening-related transcription factors in tomato is NON-RIPENING (NOR), a NAC [No apical meristem (NAM), Arabidopsis transcription activation factor (ATAF), Cup-shaped cotyledon (CUC)] domain transcription factor expressed early in ripening (Shinozaki et al., 2018). NAC genes comprise one of the largest plant-specific families of transcription factors, with specific members regulating development, defense, and senescence (Mathew and Agarwal, 2018). While all NAC genes share a conserved DNA-binding (NAC) domain, specific functional clades are defined in terms of their more variable domains, particularly the C-terminal transcriptional regulatory region. These domains can act directly as transcriptional activators, or can facilitate interaction with other transcription factors in order to fine-tune transcriptional control. NAC transcription factors have been implicated in ripening phenotypes in diverse species including tomato (Kumar et al., 2018), melon (Ríos et al., 2017), banana (Shan et al., 2012), peach (Pirona et al., 2013), and apricot (García-Gómez et al., 2019).

Apple (Malus x domestica Borkh.) fruit exhibit extensive variation in the extent and timing of ripening and softening. In previous genome-wide association studies (GWAS) of 689 apple accessions, we found a single nucleotide polymorphism (SNP) on chromosome 3 within the coding sequence of a NAC transcription factor, NAC18.1 [MD03G1222600 in the GDDH13 v1.1 reference genome (Daccord et al., 2017)], associated with harvest date and firmness (Migicovsky et al., 2016). This SNP results in an aspartate (D) to tyrosine (Y) mutation at a highly conserved position of the NAC18.1 amino acid sequence and we refer to this putatively causal SNP as D5Y. Subsequently, GWAS in several additional germplasm collections confirmed the association between the genomic region containing NAC18.1 and ripening time (Urrestarazu et al., 2017; McClure et al., 2018; Larsen et al., 2019; Jung et al., 2020). Thus, the NAC18.1 gene is a strong candidate for mediating apple ripening time and firmness, and the D5Y SNP may be of utility for marker-assisted breeding.

Due to a prolonged juvenile phase, it is particularly challenging for apple breeders to evaluate fruit quality traits. As a result, in recent years considerable effort has been invested in developing molecular markers that can be used to select for fruit quality traits at the seedling stage. In particular, markers in three genes are currently used by apple breeders to select for desirable fruit texture. The first is 1-AMINOCYCLOPROPANE1-CARBOXYLATE SYNTHASE 1 (ACS1), which encodes the ripening-associated isoform of an ethylene biosynthesis gene. The ACS1-2 allele contains a retrotransposon insertion thought to confer low ethylene production and longer shelf life in 'Fuji' and other apple cultivars homozygous for this allele (Sunako et al., 1999; Harada et al., 2000). The second gene corresponds to another enzyme involved in ethylene biosynthesis, AMINOCYCLOPROPANE-1-CARBOXYLATE OXIDASE 1 (ACO1), which has a similar reduced-functionality allele (Costa et al., 2005). Finally, POLYGALACTURONASE 1 (PG1) encodes an enzyme that hydrolyzes pectin polysaccharides in the cell wall and middle lamella and thus has been implicated in affecting apple fruit firmness (Atkinson et al., 2012). Apple firmness was identified as one of the five most important traits for genomics-assisted breeding (Laurens et al., 2018) and markers for the desirable alleles of ACS1, ACO1, and PG1 are widely available (Baumgartner et al., 2016).

The ripening of climacteric fruits, like apples, is regulated by the plant hormone ethylene (Grierson, 2013). All three firmness-related genes described above are ethylene-dependent: their expression is mediated by ethylene after the initiation of the climacteric process and is repressed by exposure to the ethylene inhibitor 1-methylcyclopropene (1-MCP) (Brummell and Harpster, 2001; Dandekari et al., 2004; Costa et al., 2005, 2010; Tadiello et al., 2016). While PG1 has been associated with apple firmness using GWAS (Kumar et al., 2013; Di Guardo et al., 2017), the discovery of ACS1, ACO1 and PG1 markers was driven largely by linkage mapping studies of bi-parental families (Costa et al., 2010; Longhi et al., 2012; Bink et al., 2014; Sadok et al., 2015). These studies did not consistently detect all three loci, which may have been due to limited sample sizes, lack of segregation between parents, and/or differences in phenotyping methods. Subsequent testing has revealed either low, or no power for these markers to predict firmness phenotypes, raising doubt about their utility in marker-assisted breeding (Costa et al., 2013; Nybom et al., 2013; McClure et al., 2018; Chagné et al., 2019).

Here we extend our previous work and report an evaluation of the role of NAC18.1 in apple ripening and softening. First, we tested the utility of the three established firmness-related markers (ACO1, ACS1 and PG1) and the NAC18.1 D5Y marker to predict harvest date, firmness and softening during storage across more than 800 diverse apple accessions. Second, we determined the effect of ethylene and 1-MCP on the expression levels of all four genes. Third, we sequenced the NAC18.1 gene from a subset of apple cultivars to discover potentially causal alleles in linkage disequilibrium (LD) with D5Y. Finally, we heterologously expressed the NAC18.1 gene in the tomato non-ripening (nor) mutant to test whether it functions as a component of a conserved fruit ripening program via complementation.

\section{MATERIALS AND METHODS}

\section{Germplasm Sources}

Apple samples taken for this study included three different sources of germplasm. The majority of the samples were 
from Canada's Apple Biodiversity Collection (ABC), an orchard located in Kentville, Nova Scotia, which contains 1,113 apple accessions. A comprehensive description of the $\mathrm{ABC}$ is provided in Watts et al. (in press). Briefly, the $\mathrm{ABC}$ is a diverse germplasm collection planted in duplicate in an incomplete block design, which includes 1 of 3 standards per grid, allowing for correction of positional effects using a REstricted Maximum Likelihood (REML) model, described in Migicovsky et al. (2017). Samples from the $\mathrm{ABC}$ were used for phenotyping of harvest date, firmness at harvest, and firmness after storage, as well as genotyping of texture-related genetic markers.

Additional apple samples were taken from the United States Department of Agriculture (USDA) apple germplasm collection and the Cornell apple breeding program in Geneva, New York. Samples from Geneva were used for sequencing of NAC18.1.

Lastly, from 2004 to 2007, 'Golden Delicious' apples from a commercial orchard in Nova Scotia were harvested before the climacteric stage, as described previously (Yang et al., 2013, 2016). Samples from this previous work were used to test the expression levels of genes of interest.

\section{Apple Phenotyping}

In 2017, we evaluated harvest date for 1,348 trees and fruit firmness for 1,328 trees within the ABC. Due to the diversity of apples within the collection, a variety of methods were used to determine the appropriate time to harvest. First, we observed if the tree had dropped fruit or, for red apples, if the fruit were a deep red color. Next, a sample apple was taken from each tree and touched to assess firmness, tasted to assess starch and sweetness, cut in half to check browning of seeds, and then sprayed with iodine solution to assess starch content. Fruit were deemed mature and ready to harvest at a starch-iodine index of 6 (Blanpied and Silsby, 1992).

Once harvested, the fruit were evaluated for firmness. We recorded the firmness $\left(\mathrm{kg} / \mathrm{cm}^{2}\right)$ of 5 fruit per tree using a penetrometer with a $1 \mathrm{~cm}$ diameter (Fruit Texture Analyzer, GS-14, Güss Manufacturing). A small section of skin was removed using a vegetable peeler, and each fruit was placed on the penetrometer platform so that the piston entered the middle of the apple where the skin had been removed. The data were automatically recorded into a spreadsheet. After these measurements were taken, 5 additional fruit from each accession (combined across trees/replicates) were placed in cold storage $\left(5^{\circ} \mathrm{C}\right)$. Fruit was removed from storage after 3 months and was left at room temperature for $24 \mathrm{~h}$ before being evaluated again for firmness using the same method. Firmness after storage and the percent change in firmness from harvest to post-storage were both calculated.

Harvesting fruit from the $\mathrm{ABC}$ orchard often lasted more than 1 day and so differences in harvest date within a week reflect the time required to harvest the orchard, rather than meaningful biological differences. As a result, we recorded harvest dates as the Monday of each week for all trees harvested throughout the week. We used the "lmer" function in the R package lme4 (Bates et al., 2015) to fit a REML model for harvest date and firmness at harvest. Next, we calculated the least squares mean using the "lsmeans" function in the lsmeans
R package (Lenth, 2016), resulting in one value per accession. After running the REML model, we had 862 unique accessions with harvest dates and 859 accessions with firmness at harvest measurements. Due to the number of fruits available and storage capacity, replicates were combined from multiple trees prior to storage and so it was not possible to fit a REML model for firmness after storage or change in firmness measurements, which both had sample sizes of 535 unique accessions. We calculated the correlation between each of the phenotypes using Pearson correlation tests and the "ggpairs" function in the GGally R package (Schloerke et al., 2020).

\section{Texture-Related Genetic Markers}

DNA was extracted from leaf tissue collected from the ABC orchard using silica columns, quantified using PicoGreen (Thermo) and normalized to a concentration of $20 \mathrm{ng} \mu \mathrm{L}^{-1}$. Genotyping was conducted using PCR and high resolution melting (HRM) on a LightScanner HR384 (BioFire). Primers are listed in Supplementary Table 1.

Genotyping of the PG1 SNP marker was based on the GenBank sequence L27743.1, where the $\mathrm{T}$ allele is favorable at position 437 and the $G$ allele is unfavorable and leads to increased softening during storage (Costa et al., 2010). Three allelic combinations of the observed indel, ACS1-1/1, 1-1/2 and 1-2/2, have been associated with high, medium, and low ethylene production, respectively (Sunako et al., 1999; Harada et al., 2000; Oraguzie et al., 2004; Costa et al., 2005). The exact position of the indel from ACS1-2 (GenBank: AB010102.1) is 1,320 to 1,483 bp (163 bp), and from ACS1-1 (GenBank: AY062129.1) is 4,500 to $4,525 \mathrm{bp}$ (25 bp). The size difference between alleles is $138 \mathrm{bp}$. The ACO1 marker involves an unfavorable $62 \mathrm{bp}$ insertion in the third intron of ACO1 (Costa et al., 2005). The third intron spans from $1,083 \mathrm{bp}$ to $1,300 \mathrm{bp}$ of the GenBank sequence Y14005.1 and the indel is found from position 1,297 bp to 1,358 bp. The D5Y mutation in NAC18.1 is a non-synonymous SNP at position $30,698,039$ on chromosome 3, according to reference genome version GDDH13 v1.1 (Daccord et al., 2017) and is associated with both harvest date and firmness (Migicovsky et al., 2016). The desirable $C$ allele encodes an aspartic acid (D) at the fifth amino acid position of the NAC18.1 protein, while the undesirable A allele encodes a tyrosine (Y). The names and IDs of samples, their phenotypes and their genotypes are provided in Supplementary Table 2 . Only accessions which were successfully genotyped for at least one of the genetic markers were included $(n=1,098)$.

\section{Phenotypic Variance Explained by Texture-Related Markers}

To determine the proportion of phenotypic variance explained by each marker of interest (ACO1, ACS1, PG1, and NAC18.1) we used a type 2 ANOVA from the 'car' package in $\mathrm{R}$ (Fox and Weisberg, 2018) with the markers encoded as co-dominant. To determine the phenotypic variance explained by each marker after accounting for harvest date, we also performed a type 2 ANOVA including the four markers and harvest date as factors. The results of the models were visualized using the "geom_tile" 
function in the ggplot2 $\mathrm{R}$ package (Wickham, 2016). We also determined the association between each marker and each phenotype using Spearman's rank correlation test. We visualized the results using the "geom_boxplot" function in ggplot2 in R (Wickham, 2016).

\section{Sequencing of NAC18.1}

DNA was isolated from leaves sampled from 18 apple accessions growing in Geneva, NY. A $2.3 \mathrm{~kb}$ amplicon including the NAC18.1 gene and $\sim 800$ bp of upstream sequence was amplified by PCR using primers NAC18F2 and NAC18R2 (Supplementary Table 1) and Phusion ${ }^{\circledR}$ High-Fidelity PCR Master Mix with HF Buffer (NEB). PCR product size and purity was confirmed by agarose gel electrophoresis, and the remaining product was purified using a DNA Clean \& Concentrator kit (Zymo Research). The resulting DNA fragment was cloned into the plasmid pMiniT 2.0 and transformed into E. coli using the $\mathrm{NEB}^{\circledR}$ PCR Cloning Kit (NEB).

Individual colonies were selected for complete sequencing of the cloned amplicon using the primers NAC18F2, NAC18F3, NAC18F4, NAC18R1, and NAC18R2 (Supplementary Table 1). For accessions homozygous for the D5Y SNP, the NAC18.1 amplicon from a single clone was sequenced. For heterozygous accessions, two clones representing each D5Y allele were selected based on partial sequencing of the D5Y region, followed by complete sequencing of the $2.3 \mathrm{~kb}$ amplicon, as described above. The nucleotide sequences were aligned using MUSCLE (Edgar, 2004) and used to construct a maximum-likelihood phylogenetic tree in MEGA7 (Kumar et al., 2016). The DNA sequence files are provided in Supplementary Table 3.

\section{Gene Expression Analyses}

Gene expression levels of ACO1, ACS1, PG1, and NAC18.1 were evaluated using q-PCR with and without treatment of ethylene and 1-methylcyclopropene (1-MCP), using methods described previously in Yang et al. (2013, 2016). Briefly, in 2004 and 2005, 'Golden Delicious' fruit were harvested from a commercial orchard before the climacteric stage. Ethylene gas $\left(36 \mu \mathrm{L} \mathrm{L}^{-1}\right)$ was applied for $24 \mathrm{~h}$ at $20^{\circ} \mathrm{C}$ to initiate ripening while control fruits were stored for $24 \mathrm{~h}$ at $20^{\circ} \mathrm{C}$ without ethylene. All fruit were then stored at $20^{\circ} \mathrm{C}$ for 21 days, with sampling occurring at day $0,7,13$, and 21 of each year. In 2006 and 2007, 'Golden Delicious' were once again harvested at the pre-climacteric stage. Apples were either treated with 1-MCP $\left(1 \mu \mathrm{L} \mathrm{L}^{-1}\right.$ of EthylBloc, $0.14 \%$, Rohm and Haas Company) for $12 \mathrm{~h}$ at $20^{\circ} \mathrm{C}$ in a sealed container, or stored at $20^{\circ} \mathrm{C}$ for $12 \mathrm{~h}$ without $1-\mathrm{MCP}$. Fruits were then stored at $20^{\circ} \mathrm{C}$, with sampling occurring on day $0,7,14$, and 22 .

Total RNA was extracted from frozen apple tissues using a hot borate method with some modification in the extraction buffer, as described in Yang et al. (2016). RNA extracts were treated with DNase I using a DNA-free Kit following the manufacturer's recommendations (Applied Biosystems). Firststrand cDNA synthesis was performed using $2 \mu \mathrm{g}$ DNase I-treated total RNA. The oligonucleotide primers used for realtime quantitative $\mathrm{qRT}$-PCR analysis were designed from sequence information in NCBI (Supplementary Table 1). Conditions for all PCR reactions were optimized as previously described (Yang et al., 2013) and efficiency values for each gene are shown in Supplementary Table 4. Two reference genes, MdActin and $M d U B I$, were used in the real-time qPCR analysis to normalize the expression patterns. Samples from day 0 (assigned an arbitrary quantity of " 1 ") were used as a calibrator to calculate relative quantities (Yang et al., 2016). The experimental design was a balanced randomized block design with random effects of year, plate, and row (on plate). The fixed effects were treatments for both ethylene [for example, control (Day 7) and ethylene (Day 7)] and 1-MCP experiments [such as control (Day 7) and 1MCP (Day 7)].

\section{Phylogenetics of NAC18.1}

To reconstruct phylogenetic relationships of NAC18.1 we mined homologous protein sequences based on genome assemblies of 17 species across the flowering plants (see Supplementary Table 5). Multiple sequence alignment was performed with MAFFT v7.450 with automatic selection of appropriate algorithm, BLOSUM62 scoring matrix, a gap open penalty of 1.26 and an offset value 0.123 (Katoh and Standley, 2013). The optimal model of protein sequence evolution for the alignment (DAYHOFF + INVGAMMA) was estimated based on AICc using Modeltest-NG v.0.1.5 on XSEDE via the CIPRES gateway (Miller et al., 2010; Darriba et al., 2020). The gene tree was reconstructed in a Bayesian framework using MrBayes v 3.2.6 (Ronquist and Huelsenbeck, 2003) implemented in Geneious Prime with two independent runs each with a chain length of 2.2 million generations; sampling every 1000th generation; 4 heated chains with a temperature of 0.2 and applying the optimal model of sequence evolution. Homologous sequence from the basal angiosperm Amborella trichopoda was used as the outgroup. After discarding the first 220,000 generations as burnin all effective sampling sizes were at least 1315, the standard deviation of clade frequencies was below 0.004 and the potential scale reduction factor (PSRF) was 1.00 suggesting appropriate convergence among the two runs.

\section{Molecular Cloning and Plant Transformation}

The hypothetical coding sequences (CDSs) corresponding to the consensus sequence of the " $\mathrm{A}$ " and " $\mathrm{C}$ " haplotypes of NAC18.1 were synthesized as a double-stranded DNA (gBlock) by Integrated DNA Technologies (IDT), with 20 bp of flanking sequences added to both ends to facilitate Gibson Assembly of the NAC18.1 sequences between the $A s c I$ and $P a c I$ restriction sites of pMDC32 (Curtis and Grossniklaus, 2003). Constructs were assembled using the NEBuilder HiFi DNA Assembly Cloning Kit (NEB) and their integrity verified by Sanger sequencing. Plasmids were transformed into Agrobacterium tumefaciens, which was used to transform tomato (S. lycopersicum) cotyledon explants (Van Eck et al., 2019) derived from the tomato nor mutant in the cv. Ailsa Craig background (LA3770, Tomato Genetics Resource Center ${ }^{1}$ ). A total of 10 T0 plants were recovered per construct and plants derived from two independent transformation events

\footnotetext{
${ }^{1}$ https://tgrc.ucdavis.edu/
} 
per construct were selected for further characterization in the $\mathrm{T} 1$ generation.

\section{Transgenic Tomato Characterization}

Fruit were harvested when visually ripe, or in the case of the nor mutant, at the equivalent age as ripe cv. Ailsa Craig fruit (the near isogenic wild-type control), as determined by tagging of flowers at anthesis. The color of the fruit surface was measured using a CR-400 Chroma Meter (Konica Minolta), and fruit were weighed, photographed, and dissected. Pericarp tissue was frozen in liquid nitrogen and stored at $-80^{\circ} \mathrm{C}$. Frozen tissue was ground to a fine powder and RNA extracted using a modified version of the protocol described in Chang et al. (1993). Briefly, approximately $400 \mathrm{mg}$ of tissue was added to a preheated $\left(80^{\circ} \mathrm{C}\right)$ two-phase system consisting of $500 \mu \mathrm{L}$ of water-saturated phenol and $500 \mu \mathrm{L}$ of extraction buffer $(100 \mathrm{mM}$ Tris [pH 8.0], $25 \mathrm{mM}$ EDTA, $2 \mathrm{M} \mathrm{NaCl}, 2 \% \mathrm{CTAB}, 2 \% \mathrm{PVP}$, and $2 \%[\mathrm{v} / \mathrm{v}]$ beta-mercaptoethanol). The mixture was vortexed and incubated for $5 \mathrm{~min}$ at $65^{\circ} \mathrm{C}$ and then cooled to room temperature before extracting and precipitating RNA, as previously described (Chang et al., 1993).

RNA was treated with RNase-free DNase (Promega) and used for cDNA synthesis with RNA to cDNA EcoDry ${ }^{\text {TM }}$ Premix with Oligo dT primer (Takara Bio). The cDNA was used as a template for quantitative PCR using Luna Universal qPCR Master Mix (NEB) and a Viia7 real-time PCR instrument (Life Technologies/ABI). Gene-specific primers are listed in Supplementary Table 1. Quantification used the $\Delta \mathrm{C}_{t}$ method with RIBOSOMAL PROTEIN L2 (RPL2) as a reference gene, and statistical significance of the $\Delta \mathrm{C}_{t}$ values was tested by a one-way ANOVA followed by Tukey's HSD test.

\section{RESULTS}

\section{Evaluation of Texture-Related Markers}

Fruit were harvested over a 65 day period and their firmness at harvest $(N=859)$ and after 3 months of cold storage $(N=535)$ was found to differ by 7 -fold across the apple accessions from the $\mathrm{ABC}$. We observed a strong relationship between harvest date and firmness: late-harvested apples were firmer both at harvest $\left(\mathrm{R}^{2}=0.25, p<1 \times 10^{-15}\right)$ and after storage $\left(\mathrm{R}^{2}=0.24\right.$, $p<1 \times 10^{-15}$ ), and they also softened less during storage $\left(\mathrm{R}^{2}=0.086, \mathrm{p}=4.53 \times 10^{-12}\right)$ (Supplementary Figure 1). Firmness at harvest was also significantly correlated with firmness after storage $\left(\mathrm{R}^{2}=0.54, p<1 \times 10^{-15}\right.$; Supplementary Figure 1). Post-harvest storage resulted in significant softening: on average, apples lost 38 percent of their firmness during 3 months of cold storage (Supplementary Table 2).

We tested the utility of four genetic markers to predict firmness-related phenotypes and found that the NAC18.1 marker outperformed the other three for both firmness at harvest and firmness after storage. However, softening (the loss in firmness during storage) was best predicted by the PG1 marker. Our results suggest that the markers in ACO1 and ACS1 have little to no predictive power for firmness-related traits across diverse apple germplasm (Figure 1; Supplementary Figure 2). When

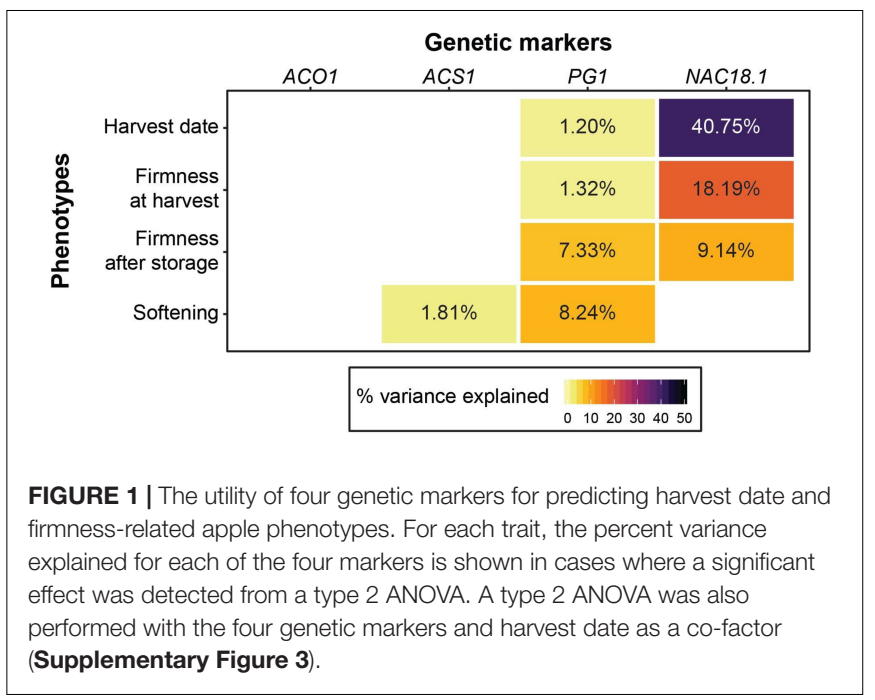

we performed the same analysis with harvest date as a factor, harvest date accounted for 10.9 and $14.6 \%$ of the variation in firmness at harvest and firmness after storage, respectively. In this model, the amount of phenotypic variance in firmness at harvest explained by NAC18.1 was reduced from $18.19 \%$, when harvest date was not included in the model, to 2.64\% (Figure 1; Supplementary Figure 3).

The genotypes of the four texture-related genetic markers across the nine most popular apple cultivars sold in the United States in 2018 (U.S. Apple Association, 2018) are presented in Figure 2. All nine cultivars were homozygous for the desirable (firm) C allele of NAC18.1, while only two to four of the cultivars had homozygous desirable genotypes for the other markers. Among the top cultivars, only 'Fuji', released in 1962, was homozygous for desirable alleles at all markers. When we examined all 904 accessions with complete genotype data across all four markers, only three possessed all four desirable genetic markers: 'Fuji,' 'Iwakami', the offspring of a 'Fuji' by 'Jonathan' cross (Yoshida et al., 1985), and 'INR 3318, an advanced breeding line from Canada. In comparison, 'McIntosh', initially discovered in 1811 and commercially released in 1870, was homozygous for undesirable alleles at all markers except for NAC18.1. 'Empire', an offspring of 'McIntosh' and 'Red Delicious' released in 1966, was homozygous for the desirable NAC18.1 alleles like its parents, but inherited undesirable alleles from 'McIntosh' and thus was heterozygous for ACS1 and ACO1, and homozygous for undesirable PG1 alleles.

Using q-PCR, we evaluated the expression of the four candidate firmness genes across a three week period and observed that transcript levels of ACO1, ACS1 and PG1 genes were upregulated in response to treatment with ethylene and suppressed following exposure to the ethylene-inhibitor, 1-MCP. NAC18.1 transcript levels, however, remained unaffected by exposure to ethylene and 1-MCP (Supplementary Figures 4, 5).

\section{Resequencing of NAC18.1}

DNA sequences from 24 NAC18.1 haplotypes confirmed the expected D5Y genotype in all individuals and revealed a 


\begin{tabular}{|c|c|c|c|c|c|}
\hline Release Date & Cultivar & ACS1 & $A C 01$ & PG1 & NAC18.1 \\
\hline 1868 & 'Granny Smith' & & & & \\
\hline 1870 & 'Mclntosh' & & & & \\
\hline 1895 & 'Red Delicious' & & & & \\
\hline 1914 & 'Golden Delicious' & & & & \\
\hline 1962 & ‘Fuji' & & & & \\
\hline 1966 & 'Empire' & & & & \\
\hline 1974 & 'Gala' & & & & \\
\hline 1990 & ‘Pink Lady®’ & & & & \\
\hline 1991 & 'Honeycrisp' & & & & \\
\hline
\end{tabular}

FIGURE 2 | Genotypes of four texture-related genetic markers across the top 9 apple cultivars sold in the United States (U.S. Apple Association). The "desirable" allele for each marker is defined as the allele that has been reported to lead to firmer apple texture.

number of additional SNPs and indels within both coding and non-coding regions of NAC18.1 (Supplementary Table 3). A multiple sequence alignment and subsequent phylogenetic analysis indicated two major clades, corresponding to the D5Y $A$ and $C$ alleles (Figure 3 ). In addition to the amino acid change resulting from D5Y, we observed several other polymorphisms

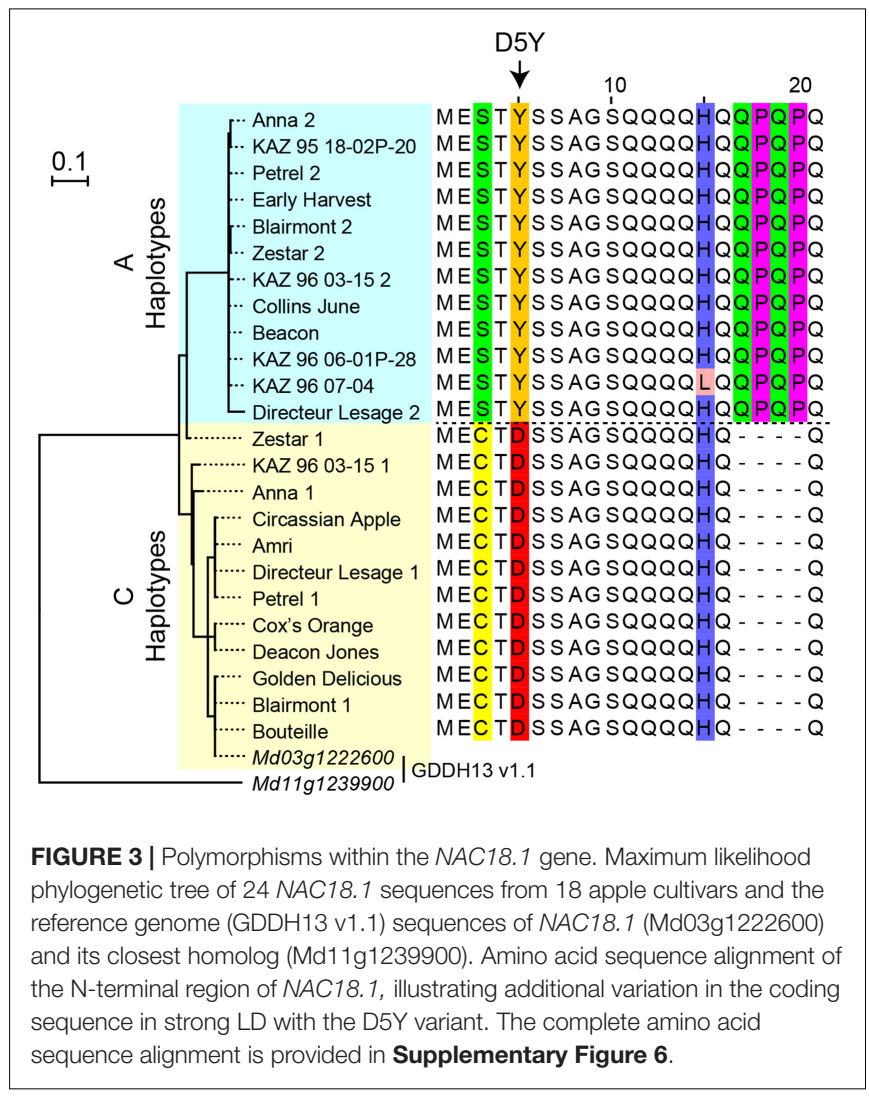

which resulted in amino acid changes. For example, near the site of the D5Y polymorphism, all "A" haplotypes also had a 12 nucleotide insertion that introduced the amino acid sequence QPQP (Figure 3; Supplementary Figure 6).

\section{Phylogenetics of NAC18.1}

NAC18.1 had 56.8\% identity with Solanum lycopersicum NOR and $57.1 \%$ and $51.7 \%$ identity with Arabidopsis thaliana NAC57 and NAC18, respectively. Arabidopsis NAC18, NAC25, and NAC56 are members of Clade III-2 as defined by Jensen et al. (2010). Protein sequences homologous to NAC18.1 were found in all sampled species except rice, suggesting gene loss within monocots. Our gene tree reconstruction revealed a gene duplication event coinciding with the ancient gamma whole-genome triplication in a common ancestor of Asterids and Rosids. This duplication resulted in a clade comprising Arabidopsis NAC25 and another clade comprising Arabidopsis NAC18 and NAC56, Solanum NOR, and Malus NAC18.1. The first clade seems to have been subsequently lost in Asterids. Within the latter clade, paralogous copies were found within the genomes of Solanum, Arabidopsis, and Malus + Pyrus, possibly related to ancient whole-genome duplication events (Ren et al., 2018). Despite these lineage-specific duplications, NAC18.1 and NOR can be considered orthologs originating from the same ancestral gene since the gamma whole-genome triplication event (Supplementary Figure 7).

\section{Transgenic Complementation of the Tomato Non-ripening (nor) Mutant}

To test whether NAC18.1 functions as a component of a conserved fruit ripening program, we introduced constructs individually conferring constitutive expression of each of the NAC18.1 haplotype CDSs into the tomato nor mutant. Two independent lines for each construct were characterized in the $\mathrm{T} 1$ generation with respect to their ability to rescue the ripening 
deficiency of the nor mutant. In contrast to nor, fruit from all four lines changed color at maturity, although internal fruit color change did not occur to the same extent as observed in a WT control (Figure 4A). To complement this qualitative phenotypic assessment, we also conducted quantitative colorimetry of the surface of the fruit (Figure 4B). Fruit from all the transgenic lines exhibited a significant increase in the $a^{*}$ (green-red) component of color space relative to the nor mutant, although only NAC18.1 ${ }^{A}$ \#6 achieved similar $a^{*}$ levels to WT.

The different degrees to which ripe fruit color was restored in the transgenic plants might be a consequence of either the different alleles of NAC18.1, or of different levels of transgene expression in each line. To address this, we analyzed the expression level of NAC18.1 using qRT-PCR primers designed to target both NAC18.1 alleles. Expression levels of the NAC18.1 transgene were not statistically different between each independent line $(p>0.5)$ (Supplementary Figure 8A). Next, we measured the expression level of several genes associated with tomato ripening physiology: PHYTOENE SYNTHASE 1 (PSY1) encodes an enzyme in an early stage of the carotenoid synthesis pathway, which is responsible for the production of red pigments during fruit ripening (Fray and Grierson, 1993), and

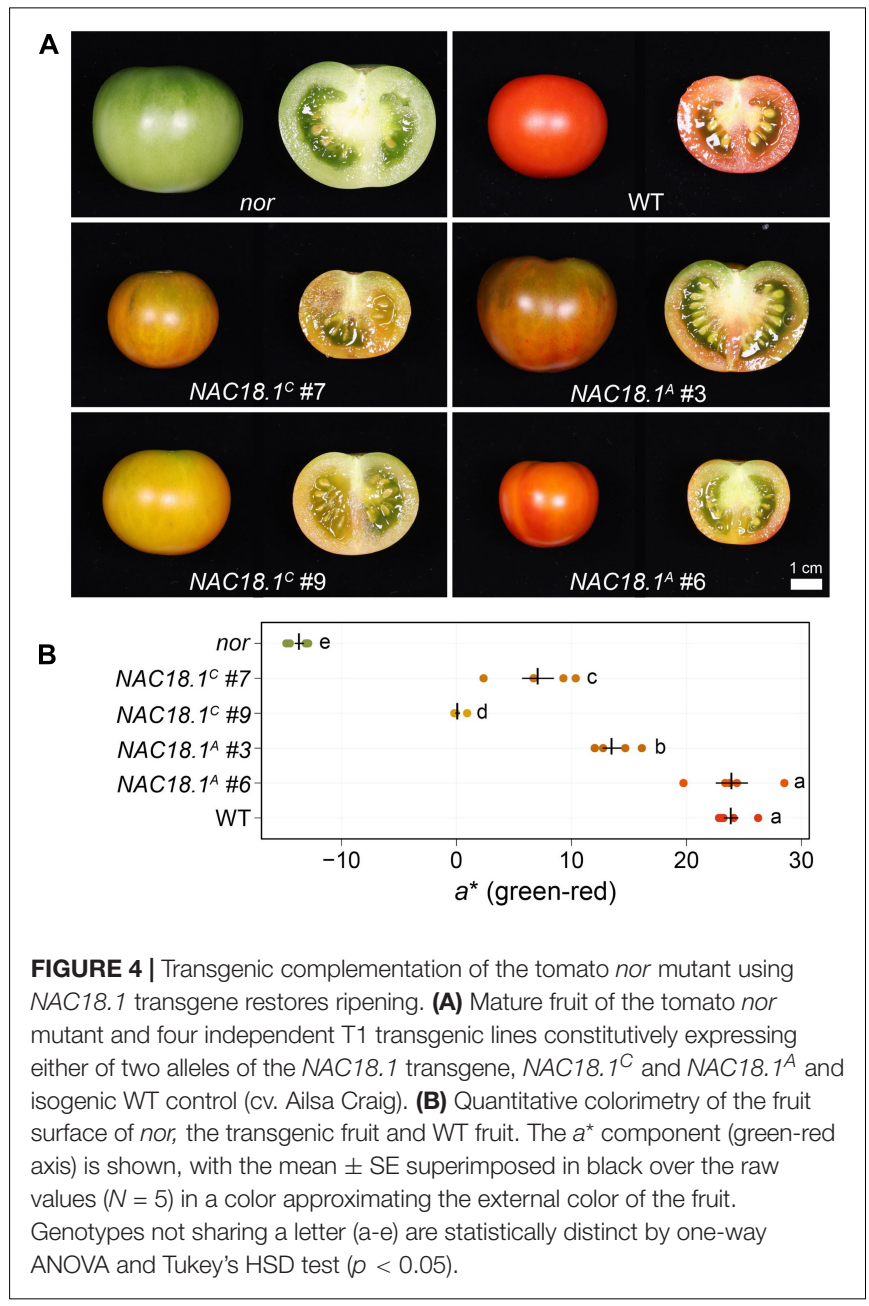

its expression is impaired in the nor mutant (Osorio et al., 2011); POLYGALACTURONASE 2 (PG2) encodes an enzyme catalyzing pectin depolymerization associated with fruit softening (Biggs and Handa, 1989); and 1-AMINOCYCLOPROPANE1-CARBOXYLATE SYNTHASE 2 (ACS2) encodes an enzyme that synthesizes 1-aminocyclopropane-1-carboxylate, the immediate precursor of ethylene (Nakatsuka et al., 1998).

Expression of all three marker genes was enhanced in the NAC18.1 transgenic lines relative to the nor mutant control, although not to the same extent as observed in WT ripe fruit (Supplementary Figures 8B-D). Visual color change was consistent with the expression of the carotenoid biosynthetic gene PSY1 (Supplementary Figure 8B). Gene expression analysis further confirmed the induction of genes involved in ripeningassociated cell wall remodeling (Supplementary Figure 8C) and ethylene synthesis (Supplementary Figure 8D). In contrast to the consistent level of NAC18.1 expression observed in each line, the marker genes were more variable in their expression levels between lines. A similar pattern was observed for all marker genes, with the NAC18.1 ${ }^{C}$ \#9 line showing the smallest induction of marker gene expression relative to nor. In the case of PG2 and ACS2, the difference in expression in NAC18.1 ${ }^{C}$ \#9 was not statistically significant relative to nor $(p=0.09$ and 0.18 , respectively). Consistent with these results, fruit from this line also exhibited the lowest amount of red color development (Supplementary Figure 8B). Taken together, these results indicate that a canonical ripening program can be induced in the tomato nor mutant through the heterologous expression of either apple NAC18.1 allele.

\section{DISCUSSION}

Genomics-assisted breeding has tremendous potential in perennial crops, such as apple, where a lengthy juvenile phase and large plant size make phenotyping at the adult stage timeconsuming and expensive. However, the genetic markers used for culling progeny at the seedling stage must accurately predict the trait of interest at the adult stage in order for genomics-assisted breeding to be effective and cost-efficient (Luby and Shaw, 2001). Apple texture has been repeatedly identified as a key breeding target because of consumer demand for crisp, firm apples that retain their desirable texture during storage (Harker et al., 2003; Yue et al., 2013; Laurens et al., 2018). Markers for three genes (ACS1, ACO1, and PG1) are widely used in genomics-assisted apple breeding programs to predict firmness. However, GWAS for firmness suggests a single major effect locus for firmness at the NAC18.1 gene (Migicovsky et al., 2016; Urrestarazu et al., 2017; McClure et al., 2018; Larsen et al., 2019). Here we have presented further evidence that whereas ACS1, ACO1 and, PG1 are weak predictors of firmness at harvest in genetically diverse apples, NAC18.1 is a strong predictor and may serve a functional role in the fruit ripening pathway.

Single-marker correlation tests between each of the four markers and four phenotypes revealed statistically significant $(p<0.05)$ associations in every case except one (Supplementary Figure 2). This result was not surprising: many genome-wide 
markers are expected to be correlated with firmness-related phenotypes since the genetic structure of our apple population is strongly correlated with these traits. For example, without correcting for the effects of population structure, $33 \%$ and $16 \%$ of genome-wide SNPs were significantly associated $(p<0.05)$ with harvest date and firmness, respectively, in the USDA collection, which is genetically identical to most of the population studied here (Migicovsky et al., 2016). Single-marker association tests do not account for population structure using genome-wide markers, as is customarily done when performing GWAS. Thus, we are only able to compare the relative power of each marker to predict these phenotypes. We found that the D5Y marker in NAC18.1 had a 3 to 14 times greater effect on firmness at harvest and harvest date than the markers in ACO1, ACS1 and PG1 (Supplementary Figure 2). We assessed the combined effects of the markers on these phenotypes using type 2 ANOVA and demonstrated that the D5Y marker in the NAC18.1 gene is a far stronger predictor of harvest date and firmness at harvest than the markers for ACS1, ACO1 and PG1. For predicting firmness after storage, however, NAC18.1 performs only slightly better than PG1, while softening during 3 months of cold storage is best predicted by the marker in PG1 (Figure 1). It is worth noting that a recent GWAS found that variation in firmness loss may not be due to variation within PG1 but rather at a neighboring ethylene response factor (ERF) gene, $E R F$ (MDP0000855671) (McClure et al., 2018). ERF may have been missed by previous linkage mapping studies due to a lack of mapping resolution compared to GWAS. Future fine mapping is required to determine if markers at ERF serve as superior predictors of softening during storage compared to the widely used PG1 marker.

In our study, by far the strongest association observed for any marker-phenotype combination was between harvest date and the D5Y marker in NAC18.1: 41\% of the variance in harvest date is accounted for by NAC18.1 (Figure 1). On average, accessions homozygous for the late-ripening NAC18.1 allele are harvested 29 days later than accessions homozygous for the early-ripening allele, which represents nearly half of the 65-day harvest season (Supplementary Figure 2). This observation is consistent with strong GWAS signals for harvest date and ripening period discovered in and around the NAC18.1 gene (Migicovsky et al., 2016; Urrestarazu et al., 2017; Larsen et al., 2019; Jung et al., 2020). The D5Y marker was also the strongest predictor of firmness at harvest and firmness after storage (Figure 1). We observed strong positive correlations between harvest date and both firmness at harvest $\left(\mathrm{R}^{2}=0.25, p<1 \times 10^{-15}\right)$ and firmness after storage $\left(\mathrm{R}^{2}=0.24, p<1 \times 10^{-15}\right.$; Supplementary Figure 1), consistent with previous work showing that earlyripening apples tend to be softer (Johnston et al., 2002; Oraguzie et al., 2004; Nybom et al., 2013). When harvest date is included as a factor in the type 2 ANOVA instead of a response variable, it is the strongest predictor of firmness both at harvest and after storage (Supplementary Figure 3). Thus, an apple's firmness is largely determined by its harvest date. Therefore, markers that predict harvest date, such as the D5Y marker in NAC18.1, will be more effective for breeding than the other firmnessrelated markers tested here. This conclusion is in agreement with previous work suggesting that screening for ACO1, ACS1 and $P G 1$ is not cost-effective and that a marker for harvest date is of greater value to improving firmness via marker-assisted breeding (Nybom et al., 2013).

The best predictor of softening during storage was the marker in PG1, which accounted for $8 \%$ of the variance. The PG1 marker was also significantly associated with firmness after storage, accounting for $7 \%$ of the variance in addition to the 9\% accounted for by NAC18.1 (Figure 1). Most apples are consumed after being stored. Thus, the most relevant phenotype for apple quality from the perspectives of both consumers and breeders is firmness after storage. The firmness of an apple after storage is a consequence of how firm it was when harvested and how much firmness loss it experienced during storage. Firmness loss, or softening, in climacteric fruit like apple is regulated by ethylene, and previous studies have shown that ACO1, ACS1 and PG1 transcripts increase as ethylene accumulates during post-harvest ripening (Costa et al., 2005, 2010; Wakasa et al., 2006; Longhi et al., 2012). We confirmed this result by showing that expression of these three genes is up-regulated by ethylene and down-regulated by the ethylene inhibitor, 1MCP (Supplementary Figures 4, 5). The expression of NAC18.1, however, was unaffected by treatments with ethylene and 1-MCP. NAC transcription factors in apple are primarily involved in the regulation of growth and development (Li et al., 2020) and previous work has suggested that ethylene may not be required for on-tree apple ripening (Lau et al., 1986; Blankenship and Unrath, 1988). Our results suggest that genetic variation at the NAC18.1 locus affects fruit firmness before harvest via a ripening pathway that is independent of ethylene, while softening during storage is ethylene-dependent and influenced by variation in or near PG1.

In the context of a marker-assisted breeding program, our results suggest that apple texture could be improved by selecting for the D5Y marker in NAC18.1. However, the potential for this marker to improve apple texture should be considered in light of several factors. First, we measured firmness with a penetrometer, but we recognize that our measures are only a proxy for the texture desired by consumers, which may be better captured using consumer panels and/or other mechanical devices that better mimic the chewing process (Costa et al., 2011). Second, all 9 of the most popular cultivars in the United States tested here are homozygous for the NAC18.1 allele associated with late-harvested, firm apples (Figure 2). Out of 1,056 accessions genotyped, 696 (66\%) are homozygous for the desirable NAC18.1 allele, and its high frequency suggests this allele may already be under selection by breeders. Indeed, a recent population genetic analysis found evidence of positive selection for the desirable NAC18.1 allele (Migicovsky et al., 2021). As a result, this allele may not segregate in many breeding populations. This may be the reason that NAC18.1 was only identified as a firmness locus via GWAS in diverse populations, and not in numerous bi-parental breeding populations, emphasizing the need for germplasm collections that maintain diverse populations (Migicovsky et al., 2019). Finally, selection for firmness using the D5Y marker will also select for late-harvested cultivars, which could result in a future excess of commercial cultivars 
with a compressed harvest window near the end of the harvest season, risking fruit loss to late season weather and reducing the opportunity to pick and sell fruit throughout the season. A more thorough understanding of the relationship between harvest date and firmness may lead to novel ways to break the correlation between these two phenotypes and to thereby enable the development of new apple cultivars with desirable firmness attributes, whose harvest dates are spread throughout the harvest season.

While the D5Y mutation in NAC18.1 is a strong functional candidate variant for apple firmness, we discovered numerous DNA sequence variants in NAC18.1, often in perfect LD with D5Y, that are also putatively functional (Figure 3). The magnitude of DNA polymorphisms at this locus, and the multitude of putatively causal variants, is consistent with the results of Larsen et al. (2019), who found 18 SNPs and 2 indels within NAC18.1 across 11 apple accessions. Our analysis of NAC18.1 sequences revealed a number of mutations that are candidates for causal association with ripening phenotypes. For example, we observed an insertion of four amino acids (QPQP) 11 amino acids downstream of the D5Y mutation in all A haplotypes upstream of the NAC DNA binding domain. Glutamine-rich sequences are common in eukaryotic transcription factors, and polymorphisms in these motifs have been shown to alter the activity of transcriptional activators (Atanesyan et al., 2012). We also observed several SNPs resulting in amino acid changes within the DNA binding domain and C-terminal transcriptional activator domain of NAC18.1. Given the pattern of LD we observed across NAC18.1 (Figure 3), there remains the possibility that the GWAS signals at NAC18.1 are driven by a causal variant outside of the NAC18.1 coding sequence that acts independently of the NAC18.1 gene. The identification of a causal polymorphism within the coding sequence or promoter of NAC18.1 will require additional high-resolution genetic mapping and/or transcriptome analysis efforts.

Since NAC18.1 is a homolog of the well-known ripening gene NOR in tomato, we used tomato as a model to explore the role of NAC18.1 in the ripening process. We observed enhanced ripening in the tomato nor mutant following heterologous expression of either of the NAC18.1 alleles (Figure 4). Although the expression level of the NAC18.1 transgene was comparable across transgenic lines (Supplementary Figure 8A), the level of ripening marker induction in the distinct lines was significantly different (Supplementary Figures 8B-D). We had hypothesized that an allele-specific trend in our transgenic experiments would indicate that coding sequence variants are responsible for the association of NAC18.1 with variation in apple ripening. While our results cannot confirm an allele-specific trend, they do verify that both alleles of NAC18.1 encode functional proteins that can promote ripening.

It is worth noting that while both NAC18.1A and NAC18.1C demonstrated an ability to rescue the ripening deficiency of the tomato nor mutant, NAC18.1A was more effective (Figure 4). Whether this reflects the modestly higher expression of NAC18.1A in the two transgenic lines as compared to the two NAC18.1C lines, or features resulting from sequence differences (e.g., protein stability or relative activity) remains unclear with available data. Nevertheless, the ability of both genes to confer recovery of ripening phenotypes in the nor mutant suggests comparable function of these genes in apple ripening. However, the cis-regulatory elements targeted by NAC18.1 in apple may differ from those targeted by NOR in tomato, and future work is required to determine if apple ripening genes targeted by NAC18.1 have the same cis-regulatory elements as their tomato orthologs.

In tomato, nor was first identified as a spontaneous mutation in an heirloom cultivar (Tigchelaar et al., 1973). While this nor allele exhibits recessive behavior and has been assumed to confer a complete loss-of-function, due to a 2 nucleotide deletion resulting in a truncated protein (Giovannoni et al., 2004), it was recently demonstrated that it is actually a dominant negative allele (Gao et al., 2019; Wang et al., 2019). These studies used CRISPR/Cas9 to generate bona fide null alleles of nor, which showed evidence of ripening relative to the spontaneous nor allele, although to a lesser extent than WT fruit. In light of these studies, we speculate that the action of the NAC18.1 transgenes was likely attenuated by the dominantnegative activity of the spontaneous nor allele used in our work. Further heterologous characterization of NAC18.1 would likely benefit from the use of null nor mutants, or double mutants of nor and nor-like 1 (Gao et al., 2018). This may allow for more precise quantitative comparisons between alleles in order to resolve whether differences in the coding sequence of NAC18.1 confer different degrees of ripening.

Although we are unable to conclude whether polymorphisms in coding or regulatory sequences affect the activity of NAC18.1 in apple, an increasing body of evidence generated using tomato as a model system indicates that coding sequence polymorphisms of NOR can influence firmness and timing of ripening. For example, the 'Alcobaça' tomato cultivar has firm fruit, delayed ripening, and long shelf life that is conferred by the alcobaça mutation, and a recent study has shown that ALCOBACA is allelic with $N O R$, and that the alcobaça allele of NOR contains a valine to aspartate mutation at position 106 within the NAC domain (Kumar et al., 2018). An additional complete loss of function allele of NOR was also found in another long shelf life tomato cultivar (Kumar et al., 2018).

In conclusion, the results presented here provide evidence that NAC18.1 is involved in apple ripening via an ethyleneindependent mechanism, and that genetic variation in and/or near the NAC18.1 gene influences apple ripening. The present study lays the groundwork for future efforts to compare the effect of different NAC18.1 haplotypes and determine the precise causal variant(s) underlying this agriculturally important gene. Ultimately, we envision that the identification of precise causal variants for ripening in apple could be exploited using gene editing for fast and efficient introgression of desirable texture phenotypes.

\section{DATA AVAILABILITY STATEMENT}

All datasets are included in the Supplementary Material. 


\section{AUTHOR CONTRIBUTIONS}

TY, JR, and JG designed the transgenic experiments. TY conducted the transgenic experiments and analyzed the results. JV provided technical support for the transgenic experiments. JS and ZZ designed the apple gene expression assays. YG conducted the apple gene expression assays. YG and JS analyzed the results. DS produced the SNP genotyping data. SW, JS, CF, $\mathrm{KB}-\mathrm{M}, \mathrm{ZM}$, and SM designed and collected the apple phenotype data. ZM analyzed the apple genotype and phenotype data. $\mathrm{RvV}$ performed the phylogenetics analysis. ZM, TY, and SM wrote the manuscript with editing assistance from JR, JS, and DS. All authors contributed to the article and approved the submitted version.

\section{FUNDING}

ZM was supported by the National Science Foundation Plant Genome Research Program 1546869. TY and JR were supported by a grant from the USDA-ARS Multistate (NE-1336) project, NYC-184821. This research was supported in part by funding the National Sciences and Engineering Research Council of Canada (SM) and A-Base funding (NOI-1238) from Agriculture and Agri-Food Canada (JS).

\section{ACKNOWLEDGMENTS}

We thank Susan Brown (Cornell University), Benjamin Gutierrez (USDA-ARS Plant Genetics Resource Unit), and C. Thomas Chao (USDA-ARS Plant Genetics Resource Unit) for apple leaf tissue samples from orchards of the Cornell apple breeding program and the USDA apple germplasm collection. Transgenic tomato plants were generated by the Boyce Thompson Institute Biotechnology Center (https://btiscience.org/). The authors gratefully acknowledge the farm services team at the Kentville Research and Development Centre for maintaining the apple collection studied here.

\section{SUPPLEMENTARY MATERIAL}

The Supplementary Material for this article can be found online at: https://www.frontiersin.org/articles/10.3389/fgene. 2021.671300/full\#supplementary-material

Supplementary Figure 1 | Correlations among phenotypes. The distributions of each phenotype are shown as well as dot plots of comparisons between each pair of phenotypes. The results of a Pearson correlation test are provided for each pairwise comparison.

\section{REFERENCES}

Atanesyan, L., Günther, V., Dichtl, B., Georgiev, O., and Schaffner, W. (2012). Polyglutamine tracts as modulators of transcriptional activation from yeast to mammals. Biol. Chem. 393, 63-70. doi: 10.1515/bc-2011-252

Atkinson, R. G., Sutherland, P. W., Johnston, S. L., Gunaseelan, K., Hallett, I. C., Mitra, D., et al. (2012). Down-regulation of POLYGALACTURONASE1
Supplementary Figure 2 | Genotype-phenotype correlations. Box plots display the variation in phenotypes across genotypic classes for four different firmness-related genetic markers. The phenotypes include (A) harvest date, (B) firmness at harvest, (C) firmness after storage, and (D) change in firmness (softening). Sample sizes are shown in parentheses under each genotypic class. The desirable genotype is on the right, while the undesirable genotype is on the left in every case. The $P$-value and rho value are shown from performing a Spearman rank correlation test. The difference in phenotype values between genotypic classes are shown across the top of each plot.

Supplementary Figure $\mathbf{3}$ | Prediction of firmness-related apple phenotypes. Four genetic markers and harvest date were included as factors in a type 2 ANOVA with three different phenotypes as outcomes. The proportion of the variance explained is shown in cases where a statistically significant result $(P<0.05)$ was observed.

Supplementary Figure 4 | Relative expression of four firmness-related genes with and without exposure to ethylene over 3 weeks of storage. The ANOVAs testing for the effects of ethylene were significant $(p<0.05)$ for ACO1 (A), ACS1 (B) and PG1 (C), but not for NAC18.1 (D). Due to the balanced design, one standard error of the means (SEM) bar is used to represent the SEM for each population.

Supplementary Figure 5 | Relative expression of four firmness-related genes with and without exposure to the ethylene-inhibitor 1-MCP over 3 weeks of storage. The ANOVAs testing for the effects of 1-MCP were significant $(p<0.05)$ for ACO1 (A), ACS1 (B) and PG1 (C), but not for NAC18.1 (D). Due to the balanced design, one standard error of the means (SEM) bar is used to represent the SEM for each population.

Supplementary Figure 6 | Amino acid sequence alignment of NAC18.1. Polymorphisms are highlighted in color. Raw sequencing data is available in Supplementary Table 3 in fasta format.

Supplementary Figure 7 | Gene tree showing phylogenetic relationships of NAC18.1. Node labels indicate posterior clade probabilities below 1; black circles indicate nodes corresponding with known ancient whole-genome multiplication events; scale bar indicates substitutions per site.

Supplementary Figure 8| Ripening marker gene expression in transgenic tomatoes. Gene expression was evaluated as the difference in threshold cycle $\left(\Delta \mathrm{C}_{\mathrm{t}}, \log _{2}\right.$ scale) by qRT-PCR using $R P L 2$ as a reference gene. The mean $\pm \mathrm{SE}$ is superimposed in black over the raw values in gray $(N=5)$. Genotypes not sharing a letter (a-d) are statistically distinct by one-way ANOVA and Tukey's HSD test $(p<0.05)$. (A) The NAC18.1 transgene. (B) PSY1, encoding the phytoene synthase carotenoid biosynthetic gene. (C) PG2, encoding the major ripening-associated polygalacturonase involved in pectin hydrolysis. (D) ACS2, encoding 1-aminocyclopropane-1-carboxylate synthase, a ripening-associated isoform of the ethylene biosynthesis enzyme.

Supplementary Table 1 | Primer names and sequences.

Supplementary Table 2 | A list of sample IDs, sample names, genotypes and phenotypes.

Supplementary Table 3 | DNA sequences from 24 NAC18. 1 haplotypes in fasta format. Amino acid sequence alignment is visualized in Supplementary Figure 6.

Supplementary Table 4 | Efficiency values for gene expression assays of apple genes ACO1, ACS1, PG1 and NAC18.1.

Supplementary Table 5 | Species and sequences sampled for phylogenetic analysis.

alters firmness, tensile strength and water loss in apple (Malus $\mathrm{x}$ domestica) fruit. BMC Plant Biol. 12:129. doi: 10.1186/1471-222912-129

Bates, D., Mächler, M., Bolker, B., and Walker, S. (2015). Fitting linear mixed-effects models Usinglme4. J. Stat. Softw. 67, 1-48. doi: 10.18637/jss.v067.i01

Baumgartner, I. O., Kellerhals, M., Costa, F., Dondini, L., Pagliarani, G., Gregori, R., et al. (2016). Development of SNP-based assays for disease resistance and fruit 
quality traits in apple (Malus $\times$ domestica Borkh.) and validation in breeding pilot studies. Tree Genet. Genomes 12:35.

Biggs, M. S., and Handa, A. K. (1989). Temporal regulation of polygalacturonase gene expression in fruits of normal, mutant, and heterozygous tomato genotypes. Plant Physiol. 89, 117-125. doi: 10.1104/pp.89.1.117

Bink, M. C. A. M., Jansen, J., Madduri, M., Voorrips, R. E., Durel, C.-E., Kouassi, A. B., et al. (2014). Bayesian QTL analyses using pedigreed families of an outcrossing species, with application to fruit firmness in apple. Theor. Appl. Genet. 127, 1073-1090. doi: 10.1007/s00122-014-2281-3

Blankenship, S. M., and Unrath, C. R. (1988). Internal ethylene levels and maturity of «Delicious» and «Golden Delicious» apples destined for prompt consumption. J. Am. Soc. Hortic. Sci. 113, 88-91.

Blanpied, G. D., and Silsby, K. J. (1992). Predicting Harvest Date Windows for Apples. Ithaca, NY: Cornell University Library.

Brummell, D. A., and Harpster, M. H. (2001). Cell wall metabolism in fruit softening and quality and its manipulation in transgenic plants. Plant Mol. Biol. 47, 311-340. doi: 10.1007/978-94-010-0668-2_18

Chagné, D., Vanderzande, S., Kirk, C., Profitt, N., Weskett, R., Gardiner, S. E., et al. (2019). Validation of SNP markers for fruit quality and disease resistance loci in apple (Malus $\times$ domestica Borkh.) using the OpenArray ${ }^{\circledR}$ platform. Hortic. Res. 6:30. doi: 10.1038/s41438-018-0114-2

Chang, S., Puryear, J., and Cairney, J. (1993). A simple and efficient method for isolating RNA from pine trees. Plant Mol. Biol. Rep. 11, 113-116. doi: 10.1007/ bf02670468

Costa, F., Cappellin, L., Longhi, S., Guerra, W., Magnago, P., Porro, D., et al. (2011). Assessment of apple (Malus $\times$ domestica Borkh.) fruit texture by a combined acoustic-mechanical profiling strategy. Postharvest Biol. Technol. 61, 21-28. doi: 10.1016/j.postharvbio.2011.02.006

Costa, F., Cappellin, L., Zini, E., Patocchi, A., Kellerhals, M., Komjanc, M., et al. (2013). QTL validation and stability for volatile organic compounds (VOCs) in apple. Plant Sci. 211, 1-7. doi: 10.1016/j.plantsci.2013.05.018

Costa, F., Peace, C. P., Stella, S., Serra, S., Musacchi, S., Bazzani, M., et al. (2010). QTL dynamics for fruit firmness and softening around an ethylene-dependent polygalacturonase gene in apple (Malusxdomestica Borkh.). J. Exp. Bot. 61, 3029-3039. doi: 10.1093/jxb/erq130

Costa, F., Stella, S., Van de Weg, W. E., Guerra, W., Cecchinel, M., Dallavia, J., et al. (2005). Role of the genes Md-ACO1 and Md-ACS1 in ethylene production and shelf life of apple (Malus domestica Borkh). Euphytica 141, 181-190. doi: 10.1007/s10681-005-6805-4

Curtis, M. D., and Grossniklaus, U. (2003). A gateway cloning vector set for highthroughput functional analysis of genes in planta. Plant Physiol. 133, 462-469. doi: 10.1104/pp.103.027979

Daccord, N., Celton, J.-M., Linsmith, G., Becker, C., Choisne, N., Schijlen, E., et al. (2017). High-quality de novo assembly of the apple genome and methylome dynamics of early fruit development. Nat. Genet. 49, 1099-1106. doi: 10.1038/ ng.3886

Dandekari, A. M., Teo, G., Defilippi, B. G., Uratsu, S. L., Passey, A. J., Kader, A. A., et al. (2004). Effect of down-regulation of ethylene biosynthesis on fruit flavor complex in apple fruit. Transgenic Res. 13, 373-384. doi: 10.1023/b: trag.0000040037.90435.45

Darriba, D., Posada, D., Kozlov, A. M., Stamatakis, A., Morel, B., and Flouri, T. (2020). ModelTest-NG: a new and scalable tool for the selection of dna and protein evolutionary models. Mol. Biol. Evol. 37, 291-294. doi: 10.1093/molbev/ msz189

Di Guardo, M., Bink, M. C. A. M., Guerra, W., Letschka, T., Lozano, L., Busatto, N., et al. (2017). Deciphering the genetic control of fruit texture in apple by multiple family-based analysis and genome-wide association. J. Exp. Bot. 68, 1451-1466. doi: $10.1093 / j x b / e r x 017$

Edgar, R. C. (2004). MUSCLE: multiple sequence alignment with high accuracy and high throughput. Nucleic Acids Res. 32, 1792-1797. doi: 10.1093/nar/gkh340

Fox, J., and Weisberg, S. (2018). An R Companion to Applied Regression. Thousand Oaks, CA: SAGE Publications.

Fray, R. G., and Grierson, D. (1993). Identification and genetic analysis of normal and mutant phytoene synthase genes of tomato by sequencing, complementation and co-suppression. Plant Mol. Biol. 22, 589-602. doi: 10. 1007/bf00047400

Gao, Y., Wei, W., Zhao, X., Tan, X., Fan, Z., Zhang, Y., et al. (2018). A NAC transcription factor, NOR-like1, is a new positive regulator of tomato fruit ripening. Hortic. Res. 5:75. doi: 10.1038/s41438-018-0111-5
Gao, Y., Zhu, N., Zhu, X., Wu, M., Jiang, C.-Z., Grierson, D., et al. (2019). Diversity and redundancy of the ripening regulatory networks revealed by the fruitENCODE and the new CRISPR/Cas9 CNR and NOR mutants. Hortic. Res. 6:39. doi: 10.1038/s41438-019-0122-x

García-Gómez, B. E., Salazar, J. A., Dondini, L., Martínez-Gómez, P., and Ruiz, D. (2019). Identification of QTLs linked to fruit quality traits in apricot (Prunus armeniaca L.) and biological validation through gene expression analysis using qPCR. Mol. Breed. 39:28. doi: 10.1007/s11032-018-0926-7

Giovannoni, J., Nguyen, C., Ampofo, B., Zhong, S., and Fei, Z. (2017). The epigenome and transcriptional dynamics of fruit ripening. Annu. Rev. Plant Biol. 68, 61-84. doi: 10.1146/annurev-arplant-042916-040906

Giovannoni, J., Tanksley, S., Vrebalov, J., and Noensie, F. (2004). Nor Gene Compositions and Methods for use Thereof. US Patent No.6762347.

Grierson, D. (2013). "Ethylene and the control of fruit ripening," in The Molecular Biology and Biochemistry of Fruit Ripening The Plant Hormone Ethylene, eds G. B. Seymour, M. Poole, J. J. Giovannoni, and G. A. Tucker (Oxford: Blackwell Publishing Ltd), 43-73. doi: 10.1002/9781118593714.ch3

Harada, T., Sunako, T., Wakasa, Y., Soejima, J., Satoh, T., and Niizeki, M. (2000). An allele of the 1-aminocyclopropane-1-carboxylate synthase gene (Md-ACS1) accounts for the low level of ethylene production in climacteric fruits of some apple cultivars. Theor. Appl. Genet. 101, 742-746. doi: 10.1007/s001220051539

Harker, F. R., Gunson, F. A., and Jaeger, S. R. (2003). The case for fruit quality: an interpretive review of consumer attitudes, and preferences for apples. Postharvest Biol. Technol. 28, 333-347. doi: 10.1016/s0925-5214(02)00215-6

Jensen, M. K., Kjaersgaard, T., Nielsen, M. M., Galberg, P., Petersen, K., O'Shea, C., et al. (2010). The Arabidopsis thaliana NAC transcription factor family: structure-function relationships and determinants of ANAC019 stress signalling. Biochem. J. 426, 183-196. doi: 10.1042/bj20091234

Johnston, J. W., Hewett, E. W., Hertog, M. L. A. T. M., and Harker, F. R. (2002). Harvest date and fruit size affect postharvest softening of apple fruit. J. Hortic. Sci. Biotechnol. 77, 355-360. doi: 10.1080/14620316.2002.11511505

Jung, M., Roth, M., Aranzana, M. J., Auwerkerken, A., Bink, M., Denancé, C., et al. (2020). The apple REFPOP-a reference population for genomics-assisted breeding in apple. Hortic. Res. 7:189.

Katoh, K., and Standley, D. M. (2013). MAFFT multiple sequence alignment software version 7: improvements in performance and usability. Mol. Biol. Evol. 30, 772-780. doi: 10.1093/molbev/mst010

Kumar, R., Tamboli, V., Sharma, R., and Sreelakshmi, Y. (2018). NAC-NOR mutations in tomato Penjar accessions attenuate multiple metabolic processes and prolong the fruit shelf life. Food Chem. 259, 234-244. doi: 10.1016/j. foodchem.2018.03.135

Kumar, S., Garrick, D. J., Bink, M. C., Whitworth, C., Chagné, D., and Volz, R. K. (2013). Novel genomic approaches unravel genetic architecture of complex traits in apple. BMC Genomics 14:393. doi: 10.1186/1471-2164-14-393

Kumar, S., Stecher, G., and Tamura, K. (2016). MEGA7: molecular evolutionary genetics analysis Version 7.0 for bigger datasets. Mol. Biol. Evol. 33, 1870-1874. doi: 10.1093/molbev/msw054

Larsen, B., Migicovsky, Z., Jeppesen, A. A., Gardner, K. M., Toldam-Andersen, T. B., Myles, S., et al. (2019). Genome-wide association studies in apple reveal loci for aroma volatiles, sugar composition, and harvest date. Plant Genome 12:180104. doi: 10.3835/plantgenome2018.12.0104

Lau, O. L., Liu, Y., and Yang, S. F. (1986). Effects of fruit detachment on ethylene biosynthesis and loss of flesh firmness, skin color, and starch in ripening Golden Delicious apples. J. Am. Soc. Hortic. Sci. 111, 731-734.

Laurens, F., Aranzana, M. J., Arus, P., Bassi, D., Bink, M., Bonany, J., et al. (2018). An integrated approach for increasing breeding efficiency in apple and peach in Europe. Hortic. Res. 5:11.

Lenth, R. V. (2016). Least-squares means: the R Packagelsmeans. J. Stat. Softw. 69, 1-33. doi: 10.18637/jss.v069.i01

Li, H., Ran, K., Dong, Q., Zhao, Q., and Shi, S. (2020). Cloning, sequencing, and expression analysis of 32 NAC transcription factors (MdNAC) in apple. PeerJ 8:e8249. doi: 10.7717/peerj.8249

Longhi, S., Moretto, M., Viola, R., Velasco, R., and Costa, F. (2012). Comprehensive QTL mapping survey dissects the complex fruit texture physiology in apple (Malus x domestica Borkh.). J. Exp. Bot. 63, 1107-1121. doi: 10.1093/jxb/err326

Lü, P., Yu, S., Zhu, N., Chen, Y.-R., Zhou, B., Pan, Y., et al. (2018). Genome encode analyses reveal the basis of convergent evolution of fleshy fruit ripening. Nat. Plants 4, 784-791. doi: 10.1038/s41477-018-0249-z 
Luby, J. J., and Shaw, D. V. (2001). Does marker-assisted selection make dollars and sense in a fruit breeding program? HortScience 36, 872-879. doi: 10.21273/ hortsci.36.5.872

Mathew, I. E., and Agarwal, P. (2018). May the fittest protein evolve: favoring the plant-specific origin and expansion of NAC transcription factors. BioEssays 40:1800018. doi: 10.1002/bies.201800018

McClure, K. A., Gardner, K. M., Douglas, G. M., Song, J., Forney, C. F., DeLong, J., et al. (2018). A genome-wide association study of apple quality and scab resistance. Plant Genome 11, 1-14. doi: 10.3835/plantgenome2017.08.0075

Migicovsky, Z., Gardner, K. M., Richards, C., Thomas Chao, C., Schwaninger, H. R., Fazio, G., et al. (2021). Genomic consequences of apple improvement. Hortic. Res. 8:9.

Migicovsky, Z., Li, M., Chitwood, D. H., and Myles, S. (2017). Morphometrics reveals complex and heritable apple leaf shapes. Front. Plant Sci. 8:2185. doi: 10.3389/fpls.2017.02185

Migicovsky, Z., Warschefsky, E., Klein, L. L., and Miller, A. J. (2019). Using living germplasm collections to characterize, improve, and conserve woody perennials. Crop. Sci. 59, 2365-2380. doi: 10.2135/cropsci2019.05.0353

Migicovsky, Z., Gardner, K. M., Money, D., Sawler, J., Bloom, J. S., Moffett, P., et al. (2016). Genome to phenome mapping in apple using historical data. Plant Genome 9. doi: 10.3835/plantgenome2015.11.0113

Miller, M. A., Pfeiffer, W., and Schwartz, T. (2010). "Creating the CIPRES Science Gateway for inference of large phylogenetic trees," in Gateway Computing Environments Workshop (GCE), 14 Nov. 2010, New Orleans, LA, 1-8.

Nakatsuka, A., Murachi, S., Okunishi, H., Shiomi, S., Nakano, R., Kubo, Y., et al. (1998). Differential expression and internal feedback regulation of 1-Aminocyclopropane-1-Carboxylate Synthase, 1-Aminocyclopropane-1Carboxylate oxidase, and ethylene receptor genes in tomato fruit during development and ripening. Plant Physiol. 118, 1295-1305. doi: 10.1104/pp.118. 4.1295

Nybom, H., Ahmadi-Afzadi, M., Sehic, J., and Hertog, M. (2013). DNA markerassisted evaluation of fruit firmness at harvest and post-harvest fruit softening in a diverse apple germplasm. Tree Genet. Genomes 9, 279-290. doi: 10.1007/ s11295-012-0554-z

Oraguzie, N. C., Iwanami, H., Soejima, J., Harada, T., and Hall, A. (2004). Inheritance of the Md-ACS1 gene and its relationship to fruit softening in apple (Malus $\times$ domestica Borkh.). Theor. Appl. Genet. 108, 1526-1533. doi: 10.1007/s00122-003-1574-8

Osorio, S., Alba, R., Damasceno, C. M. B., Lopez-Casado, G., Lohse, M., Zanor, M. I., et al. (2011). Systems biology of tomato fruit development: combined transcript, protein, and metabolite analysis of tomato transcription factor (nor, rin) and ethylene receptor $(\mathrm{Nr})$ mutants reveals novel regulatory interactions. Plant Physiol. 157, 405-425. doi: 10.1104/pp.111.175463

Pirona, R., Eduardo, I., Pacheco, I., Da Silva Linge, C., Miculan, M., Verde, I., et al. (2013). Fine mapping and identification of a candidate gene for a major locus controlling maturity date in peach. BMC Plant Biol. 13:166. doi: 10.1186/14712229-13-166

Ren, R., Wang, H., Guo, C., Zhang, N., Zeng, L., Chen, Y., et al. (2018). Widespread whole genome duplications contribute to genome complexity and species diversity in angiosperms. Mol. Plant 11, 414-428. doi: 10.1016/j.molp.2018.01. 002

Ríos, P., Argyris, J., Vegas, J., Leida, C., Kenigswald, M., Tzuri, G., et al. (2017). ETHQV6.3 is involved in melon climacteric fruit ripening and is encoded by a NAC domain transcription factor. Plant J. 91, 671-683. doi: 10.1111/tpj.13596

Ronquist, F., and Huelsenbeck, J. P. (2003). MrBayes 3: bayesian phylogenetic inference under mixed models. Bioinformatics 19, 1572-1574. doi: 10.1093/ bioinformatics/btg180

Sadok, I. B., Ben Sadok, I., Tiecher, A., Galvez-Lopez, D., Lahaye, M., LasserreZuber, P., et al. (2015). Apple fruit texture QTLs: year and cold storage effects on sensory and instrumental traits. Tree Genet. Genomes 11:119. doi: 10.1007/ s11295-015-0947-x

Schloerke, B., Crowley, J., Cook, D., Briatte, F., Marbach, M., Thoen, E., et al. (2020). GGally: Extension to "ggplot2.". Available online at: https://cran.rproject.org/web/packages/GGally/index.html.

Shan, W., Kuang, J.-F., Chen, L., Xie, H., Peng, H.-H., Xiao, Y.-Y., et al. (2012). Molecular characterization of banana NAC transcription factors and their interactions with ethylene signalling component EIL during fruit ripening. J. Exp. Bot. 63, 5171-5187. doi: 10.1093/jxb/ers178
Shinozaki, Y., Nicolas, P., Fernandez-Pozo, N., Ma, Q., Evanich, D. J., Shi, Y., et al. (2018). High-resolution spatiotemporal transcriptome mapping of tomato fruit development and ripening. Nat. Commun. 9:364.

Sunako, T., Sakuraba, W., Senda, M., Akada, S., Ishikawa, R., Niizeki, M., et al. (1999). An allele of the ripening-specific 1-Aminocyclopropane-1-Carboxylic Acid Synthase Gene (ACS1) in apple fruit with a long storage life. Plant Physiol. 119, 1297-1304. doi: 10.1104/pp.119.4.1297

Tadiello, A., Longhi, S., Moretto, M., Ferrarini, A., Tononi, P., Farneti, B., et al. (2016). Interference with ethylene perception at receptor level sheds light on auxin and transcriptional circuits associated with the climacteric ripening of apple fruit (Malus x domestica Borkh.). Plant J. 88, 963-975. doi: 10.1111/tpj. 13306

Tigchelaar, E. C., Tomes, M. L., Kerr, E. A., and Barman, R. J. (1973). A new fruit ripening mutant, non-ripening (nor). Rep. Tomato Genet. Coop. Tomato Genet. Coop. 23, 33-34.

U.S. Apple Association. (2018). Apple Industry At-a-Glance. Available online at: http://usapple.org/the-industry/apple-industry-at-a-glance/(accessed February 11, 2018).

Urrestarazu, J., Muranty, H., Denancé, C., Leforestier, D., Ravon, E., Guyader, A., et al. (2017). Genome-wide association mapping of flowering and ripening periods in apple. Front. Plant Sci. 8:1923. doi: 10.3389/fpls.2017.01923

Van Eck, J., Keen, P., and Tjahjadi, M. (2019). "Agrobacterium tumefaciensMediated Transformation of Tomato," in Methods in Molecular Biology, eds S. Kumar, P. Barone, and M. Smith (New York, NY: Humana Press), 225-234. doi: 10.1007/978-1-4939-8778-8_16

Wakasa, Y., Kudo, H., Ishikawa, R., Akada, S., Senda, M., Niizeki, M., et al. (2006). Low expression of an endopolygalacturonase gene in apple fruit with longterm storage potential. Postharvest Biol. Technol. 39, 193-198. doi: 10.1016/j. postharvbio.2005.10.005

Wang, D., Yeats, T. H., Uluisik, S., Rose, J. K. C., and Seymour, G. B. (2018). Fruit Softening: revisiting the role of pectin. Trends Plant Sci. 23, 302-310. doi: 10.1016/j.tplants.2018.01.006

Wang, R., da Rocha Tavano, E. C., Lammers, M., Martinelli, A. P., Angenent, G. C., and de Maagd, R. A. (2019). Re-evaluation of transcription factor function in tomato fruit development and ripening with CRISPR/Cas9-mutagenesis. Sci. Rep. 9:1696. doi: 10.1038/s41598-018-38170-6

Watts, S., Migicovsky, Z., McClure, K., Yu, H., Amyotte, B., Baker, T., et al. (in press). Quantifying apple diversity: a phenomic characterization of Canada's Apple Biodiversity Collection. Plants, People, Planet. doi: 10.1002/ppp3.10211

Wickham, H. (2016). ggplot2: Elegant Graphics for Data Analysis. Berlin: Springer Science \& Business Media.

Yang, X., Song, J., Campbell-Palmer, L., Fillmore, S., and Zhang, Z. (2013). Effect of ethylene and 1-MCP on expression of genes involved in ethylene biosynthesis and perception during ripening of apple fruit. Postharvest Biol. Technol. 78, 55-66. doi: 10.1016/j.postharvbio.2012.11.012

Yang, X., Song, J., Du, L., Forney, C., Campbell-Palmer, L., Fillmore, S., et al. (2016). Ethylene and 1-MCP regulate major volatile biosynthetic pathways in apple fruit. Food Chem. 194, 325-336. doi: 10.1016/j.foodchem.2015. 08.018

Yoshida, Y., Haniuda, T., Tsuchiya, S., Sanada, T., Masuda, T., Bessho, H., et al. (1985). New apple cultivar iwakami. Bull. Fruit Tree Res. Stat. Ser. C 12, 11-20.

Yue, C., Karina Gallardo, R., Luby, J., Rihn, A., McFerson, J. R., McCracken, V., et al. (2013). An Investigation of U.S. Apple Producers' Trait PrioritizationEvidence from audience surveys. HortScience 48, 1378-1384. doi: 10.21273/ hortsci.48.11.1378

Conflict of Interest: The authors declare that the research was conducted in the absence of any commercial or financial relationships that could be construed as a potential conflict of interest.

Copyright (๑) 2021 Migicovsky, Yeats, Watts, Song, Forney, Burgher-MacLellan, Somers, Gong, Zhang, Vrebalov, van Velzen, Giovannoni, Rose and Myles. This is an open-access article distributed under the terms of the Creative Commons Attribution License (CC BY). The use, distribution or reproduction in other forums is permitted, provided the original author(s) and the copyright owner(s) are credited and that the original publication in this journal is cited, in accordance with accepted academic practice. No use, distribution or reproduction is permitted which does not comply with these terms. 